University of Nebraska - Lincoln

DigitalCommons@University of Nebraska - Lincoln

1995

\title{
The effects of extra vehicular activity (EVA) gloves on human performance
}

Ram R. Bishu

University of Nebraska-Lincoln, RBISHU1@UNL.EDU

Glenn Klute

NASA

Follow this and additional works at: https://digitalcommons.unl.edu/imsefacpub

Bishu, Ram R. and Klute, Glenn, "The effects of extra vehicular activity (EVA) gloves on human performance" (1995). Industrial and Management Systems Engineering Faculty Publications. 75. https://digitalcommons.unl.edu/imsefacpub/75

This Article is brought to you for free and open access by the Industrial and Management Systems Engineering at DigitalCommons@University of Nebraska - Lincoln. It has been accepted for inclusion in Industrial and Management Systems Engineering Faculty Publications by an authorized administrator of DigitalCommons@University of Nebraska - Lincoln. 


\title{
The effects of extra vehicular activity (EVA) gloves on human performance
}

\author{
Ram R. Bishu ${ }^{\mathrm{a}, *}$, Glenn Klute ${ }^{\mathrm{b}}$ \\ a Department of Industrial Engineering, Unicersity of Nebraska, Lincoln, NE 68588-0518. USA \\ b Anthropometry and Biomechanics Laboratory, NASA, Johnson Space Center, Houston, TX, USA
}

Received 30 June 1993; revised 5 August 1994

\begin{abstract}
Human strength and capabilities such as dexterity, manipulability, and tactile perception are unique and render the hand as a very versatile, effective, multipurpose tool. This is especially true for unknown microgravity environments such as the EVA environment. Facilitation of these activities, with simultaneous protection from the cruel EVA environment, are the two, often conflicting, objectives of glove design. The objective of this study was to assess the effects of EVA gloves at different pressures on human hand capabilities. A factorial experiment was performed in which three types of EVA gloves were tested at five pressure differentials. The independent variables tested in this experiment were gender, glove type, pressure differential, and glove make. Six subjects participated in an experiment in which a number of dexterity measures such as time to tie a rope, and the time to assemble a nut and bolt, were recorded. Tactility was measured through a two-point discrimination test. The results indicate that (a) With EVA gloves there is a considerable reduction in both strength and dexterity performance; and (b) performance decrements increase with increasing pressure differential. Some interesting gender glove interactions were observed, some of which may have been due to the extent (or lack of) fit of the glove to the hand. The implications for the designer are discussed.
\end{abstract}

\section{Relevance to industry}

Gloves are used almost in all the industries as a safety device to protect the human hand. However, performance is reduced with gloves. This study attempts to link glove attributes to performance of gloved hand. Hence it is very relevant to the industry.

Keywords: Strength; Dexterity; Tactility; Gloves

This work has been presented in parts at the Interna tional Ergonomics and Safety Conference held in Copenhagen, Denmark in June 1993, and at the Annual Conference of the Human Factors and Ergonomics Society held in Seattle, USA in October 1993.

* Corresponding author.

\section{Introduction}

Human capabilities such as dexterity, manipulability, and tactile perception are unique and render the hand as a very versatile, effective, multipurpose tool. This is especially true for EVA microgravity environment. Under these condi- 
tions the hand becomes the primary means of locomotion, restraint and material handling. Facilitation of these activities, with simultaneous protection from the hazards of the EVA environment, are often conflicting objectives of glove design. The conflicts associated with providing primary hand protection through the use of a glove while permitting adequate hand functioning have been widely recognized.

Numerous articles have been published in the area of the effect of gloves on task performance. Lyman and Groth (1958) reported that when gloves were worn, subjects exerted more force than when barehanded while inserting pins into a pegbox. Bradley (1969) studied the operation time of five types of control tasks with bare hand, wool gloves, and leather over wool gloves. The results of his research showed that the operation time depends on the type of gloves, the type of control operations, and the physical characteristics of the controls. Cochran et al. (1986) studied grasp force degradation of some commercially available gloves. Five types of gloves and bare hand conditions were compared and the results showed that all the gloves tested reduced the maximum grasp force significantly when compared to bare hand condition. Wang et al. (1987) also found similar results. The basic overall findings of these studies are (a) gloves reduce strength capabilities, and (b) gloves reduce dexterity and manipulability.

While most of the studies have addressed performance compromises with commercial gloves, very few studies have attempted to assess the effects of EVA gloves on basic hand capabilities. Perhaps the most comprehensive study performed on the assessment of performance decrements with EVA gloves is the one done by O'Hara et al. (1988). The authors had studied two levels of hand conditions (gloved and barehanded), two levels of pressure differential ( 0 psid, and 4.3 psid), and three levels of hand size (small, medium, and large). The salient findings were: (a) Gloves reduced basic hand grip strength, and the pressure differential reduced it further; however, neither the glove nor the pressure had any effect on pinch strength; (b) the degradation in tactile perception was more noticeable with glove use than with pressure change; and (c) dexterity was reduced by both glove and pressure. Unpressurized glove reduced dexterity by $50 \%$, while pressurizing reduced it further by $30 \%$.

The rationale for this investigation evolved out of the above study. The O'Hara (1988) investigation used one type of glove and one pressure level. It is recognized that in EVA tasks the prebreath time before donning the suit is a function of the pressure. Prebreathing is an activity performed prior to donning the space suit for EVA activities. The purpose of prebreathing is to let the body achieve new physiological homeostasis for activities at new, lower pressure. The greater the pressure, the shorter the prebreathing time. However, the performance decrement is also a function of pressure, with larger decrements at greater pressure. An important piece of information that is needed, and which is currently unavailable, is the pressure performance profile for the various EVA gloves. Therefore, the objective was to develop functional relations between performance decrements and pressure differential for EVA gloves.

\section{Methods}

\subsection{Subjects}

Six subjects (three males and three females) participated in this experiment. Their participation was voluntary.

\subsection{Independent variables}

The independent variables tested in this experiment were gender, glove type, pressure differential, and glove configurations. The six subjects were equally split between two genders to provide the gender differences. Two types of glove assembly were used: with and without thermal micrometeorite garment (TMG). An EVA glove is an assemblage of two major units: an inner pressurizing glove and an outer TMG glove. One of the objectives was to assess the exact effect of TMG on performance. Current Shuttle gloves operate at 4.3 psid. Certain developmental gloves are being designed to operate at 8.3 psid. The 
rationale being that operating at higher pressure differentials results in the pre-breathing time being reduced considerably. Five levels of pressure differentials were used in this experiment: 0 psid, 3.2 psid, 4.3 psid, 6.3 psid, and 8.3 psid. The intent was to develop a pressure-performance decrement profile. Three different glove configurations were tested here: current Shuttle 3000 series WETF training gloves (referred to hereafter as GLOVE C), and two advanced developmental gloves (referred to hereafter as GLOVES $A$ and $B$ ). To summarize, the independent variables with their respective levels were:
(1) Gender
male and female
(2) Glove type with and without TMG
(3) Pressure
$0,3.2,4.3,6.3,8.3$
(4) Glove configuration
$\mathrm{A}, \mathrm{B}$, and $\mathrm{C}$

\subsection{Performance measures}

The performance measures were selected based on the O'Hara (1988) study, and comprised two strength measures (grip and pulp pinch strength), two dexterity measures (nuts-bolts test and rope tying test), and a tactility measure (twopoint discrimination test). The criteria for selection of performance measures were (a) they should be generic, and hence repeatable, and (b) they should be reasonably representative of the EVA activities. The grip strength was measured by a standard JAMAR hand dynamometer. The dynamometer was wired to a digital display, which gave the grip strength readings in pounds. The grip span of the hand dynamometer was kept constant throughout the experiment at 2 inches. The pinch strength was measured by a B\&L $(60$ pounds) pinch gauge. Dexterity and manipulability were measured by the rope-tying test and the nuts-bolts test. The former consisted of pushing a rope through a hole on a wooden panel and tying a shoe lace knot around the panel. In order to gauge the size effect, rope of three sizes (small, medium, and large) was used. The time to tie the knot was recorded as a performance measure. The wooden panel had three holes through which three pairs of nuts and bolts (small, medium, and large) were assembled. The nuts and bolts assembly task consisted of undoing the nut from the assembly, showing the nut and bolt to the test experimenter, and reassembling the nut and bolt on the wooden panel. The mean assembly time was recorded and used as a measure of dexterity. The final measure recorded in this experiment was the performance in the modified two-point discrimination test. O'Hara et al. (1988) had used a modified version of a two-point discrimination (2PD) test for assessing the tactile sensitivity of subjects under different test conditions. A similar apparatus was fabricated here to measure the tactile sensitivity. In essence the apparatus consisted of a " $V$ " block through which the subjects had to slide their fingers. The " $V$ " block was graduated, and the distance from the starting end to the point where the subjects could feel two edges was treated as the tactility score. As the force with which the subjects could press the "V" block was an uncontrollable variable which could influence the results, the " $V$ " block design used by O'Hara et al. (1988) was modified to have a balancing weight on the underside of the apparatus. The dead weight was expected to facilitate constant application of force on the " $V$ " block during the administration of the two-point discrimination test.

\subsection{Glove box}

The testing was done in Advanced Suit Laboratory. The actual tests were conducted inside a glove box (Fig. 1). The glove box is cylindrical in shape, approximately $2 \mathrm{ft}$ in diameter and $4 \mathrm{ft}$ in length with an internal volume of $13 \mathrm{ft}^{3}$. On each side of the glove box were two end caps, made of Plexiglas and bolted through 8 bolts. About midway along the axis of the glove box were 2 six in. circular openings in the cylinder wall, placed shoulder width apart, which provided access and attachment point for the EVA glove and arm assemblies. The glove box was connected to a vacuum pump and could be evacuated to the desired pressure level. There was a gauge on the outer cylinder wall calibrated to read the pressure differential. 


\subsection{Procedure}

The levels of independent variables were factorially combined to yield 26 experimental conditions. There were 26 experimental conditions in this experiment (see Table 1). The order of presentation of these was randomized for each subject. In addition, all the subjects performed a 'Bare-handed' condition on the last day. Within a condition the order of presentation of the five tasks (grip, pinch, nuts-bolts, rope tying, and 2PD test) was also randomized for each subject. As stated earlier six subjects participated in this study. Gender was a between-subject factor. Each subject performed one condition per day, resulting in 26 days of experimentation in all. A trial consisted of the following steps.
Table 1

Experimental design

\begin{tabular}{|c|c|c|c|c|c|c|}
\hline Pressure & A & $\begin{array}{l}\text { A with } \\
\text { TMG }\end{array}$ & B & $\begin{array}{l}\text { B with } \\
\text { TMG }\end{array}$ & $\mathrm{C}$ & $\begin{array}{l}\text { C with } \\
\text { TMG }\end{array}$ \\
\hline 0 psid & & & & & & \\
\hline $\begin{array}{l}3.2 \\
\text { psid }\end{array}$ & $\nu$ & 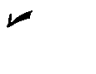 & 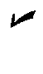 & $r$ & $\nu$ & $r$ \\
\hline $\begin{array}{l}4.3 \\
\text { psid }\end{array}$ & $\nu$ & $\sim$ & $r$ & $\nu$ & $\nu$ & $r$ \\
\hline $\begin{array}{l}6.3 \\
\text { psid }\end{array}$ & $w$ & $w$ & $\nu$ & $\nu$ & NA & $\mathrm{NA}$ \\
\hline $\begin{array}{l}8.3 \\
\text { psid }\end{array}$ & $r$ & $\nu$ & $\nu$ & & $N A$ & $\mathrm{NA}$ \\
\hline
\end{tabular}

(1) The glove box was pressurized to the required level.

(2) The subject donned a pair of comfort gloves and the EVA gloves for that day's trial.

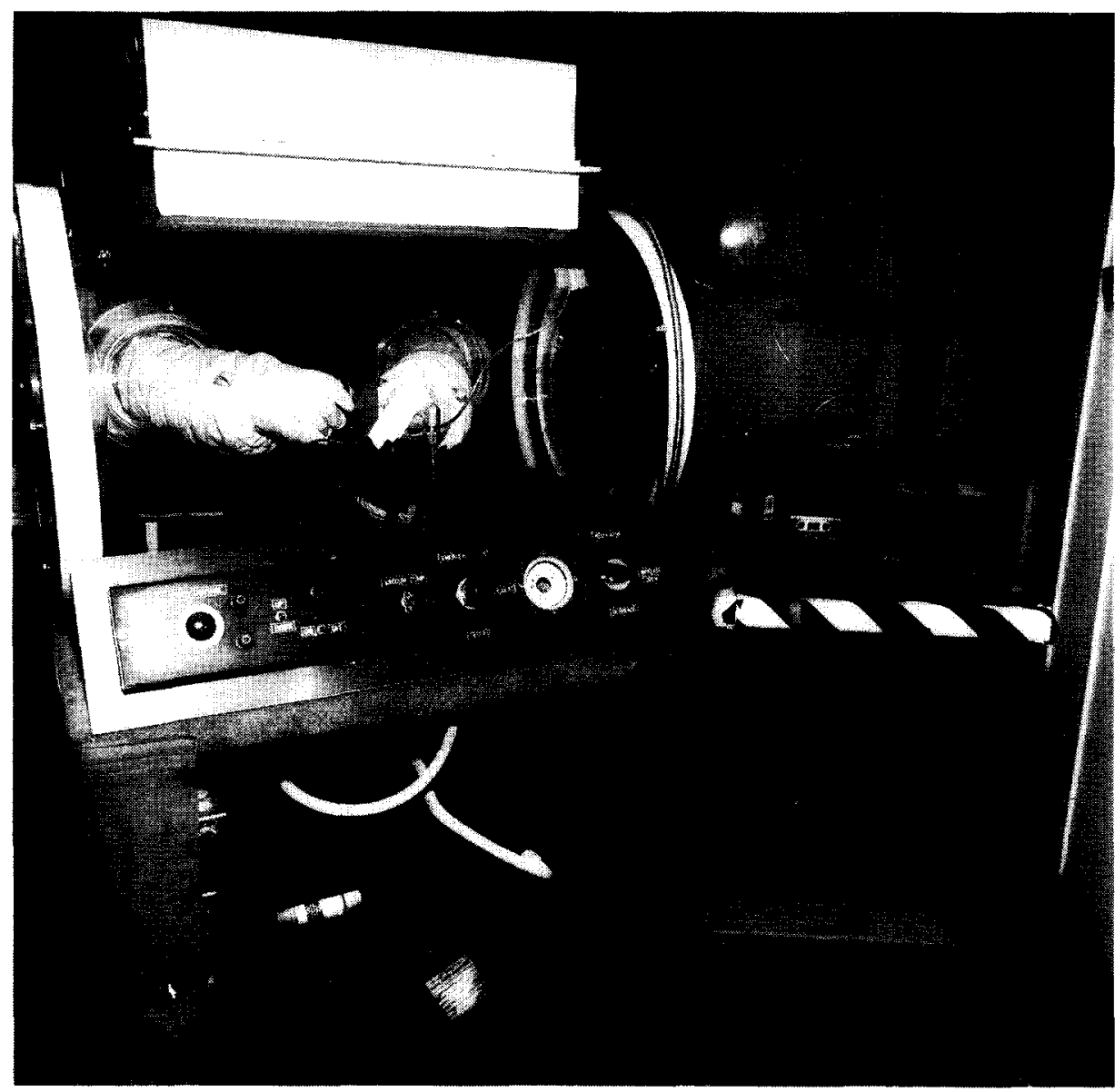

Fig. 1. Experimental set-up 
(3) Grip strength was recorded through a Jamar Hand Dynamometer connected to a digital display and to a Teac Recorder.

(4) Pulp pinch strength was measured following a 2-minute rest period using a pinch gauge.

(5) For the nuts-bolts test, three pairs of nuts and bolts (large, medium, and small size) were mounted on a wooden panel. The task involved removing the nut from its respective bolt, and mounting the nut back again. The time for this activity was recorded with a stop watch.

(6) The rope-tying test consisted of tying a simple shoe lace knot on the same wooden panel that had the nuts and bolts. Three sizes of ropes (small, medium, and large) were used and the time to tie was recorded with a stop watch.

(7) 2 PD test consisted of the subjects sliding their right index finger along the edges of the "V block". The distance of the point at which they felt two edges from their starting point was recorded as their tactility score. In order to keep the force at the point of contact constant the "V block" had a balancing weight on the other side.

Fig. 1 shows the sketch of the experimental set-up with nuts-bolts panel. Fig. 2 shows the sketch of the three gloves tested. A trial lasted for about 20 minutes. Further details of this procedure are described in Bishu and Klute (1993).

For purposes of clarity the data was analyzed first with strength as dependent measure, and then with dexterity measures as dependent variables.

\section{Results}

This study had a number of performance measures. The results will be presented under two headings: strength as performance measure and dexterity as performance measures.

\subsection{Strength as dependent variable}

The data on grip strength and pinch strength was subjected to an analysis of variance (ANOVA) and Table 2 shows the ANOVA summary. It is seen that all the main factors are significant for pulp pinch strength, while glove type (TMG or NO TMG) effect is not significant for grip strength. Female subjects exhibited lower strengths than their male counterparts as shown in Table 3, which shows the average strength across the three pairs of gloves tested. This result is consistent with the general findings that female strength capabilities are about $60-70 \%$ of male capabilities. Fig. 3 shows the plot of Glove effect on strength. Compared to bare hand capabilities, there is a $50 \%$ reduction in grip strength when gloves are donned. The corresponding reduction in pinch strength is very small (approximately $10 \%$ ). The gender difference is somewhat consistent across all the gloves. Fig. 4 shows the plot of

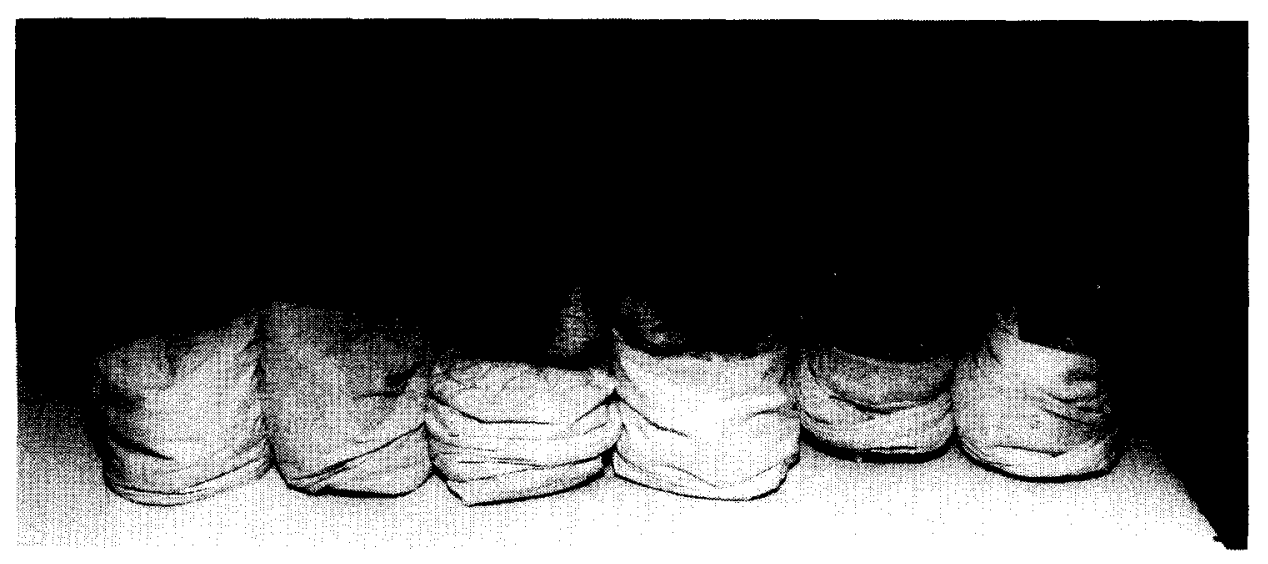

Fig. 2. Different types of gloves test. 
Table 2

ANOVA summary on grip and pinch strength

\begin{tabular}{lllllllllll}
\hline Dep. var & Gender & $\begin{array}{l}\text { Glove } \\
\text { make }\end{array}$ & $\begin{array}{l}\text { Glove } \\
\text { type }\end{array}$ & Pressure & $\begin{array}{l}\text { Type } * \\
\text { make }\end{array}$ & $\begin{array}{l}\text { Make } * \\
\text { pressure }\end{array}$ & $\begin{array}{l}\text { Type } * \\
\text { pressure }\end{array}$ & $\begin{array}{l}\text { Gender } * \\
\text { make }\end{array}$ & $\begin{array}{l}\text { Gender } * \\
\text { type }\end{array}$ & $\begin{array}{l}\text { Gender } * \\
\text { pressure }\end{array}$ \\
\hline Grip & $* * *$ & $*_{* *}$ & $\mathrm{~ns}$ & $* * *$ & $* *$ & $* * *$ & $*$ & $\mathrm{~ns}$ & ${ }^{* * *}$ & $\mathrm{~ns}$ \\
Pinch & $* * *$ & $*_{* *}$ & $* * *$ & $* * *$ & $\mathrm{~ns}$ & $\mathrm{n}$ & $*$ & $n_{* *}$ & $\mathrm{~ns}$ & $* * *$ \\
\hline
\end{tabular}

$\overline{{ }^{* * *}{ }^{*} p}<0.0001 ;{ }^{*}{ }^{*} p<0.001 ;{ }^{*} p<0.01$

Table 3

Gender effect on grip and pinch strengths

\begin{tabular}{lll}
\hline Strength & Male & Female \\
\hline Grip & $58.84(18.57)$ lbs. & $36.93(11.75)$ lbs. \\
Pinch & $17.94(5.30)$ lbs. & $13.08(2.79)$ lbs. \\
\hline
\end{tabular}

(Standard deviation in parentheses)

pressure effect on strength. The most interesting aspect of Fig. 4 is that the gender differences appear to disappear at higher pressure differentials. As expected, performance reduces with increasing pressure differential. Strength reductions are large from bare hand to glove condition. It appears that there are two levels of performance decrements with pressure. Performance at 3.2 and 4.3 psi look similar while performance at 6.3 and 8.3 psi appear similar, and worse than other pressure differentials.

Fig. 5 shows the plot of Glove * TMG interaction. Glove $\mathrm{C}$ seems to stand out from the other two. TMG seems to reduce strength on Glove C, while the opposite effect is observed on gloves $\mathrm{A}$, and B. A Gender * TMG interaction appears to

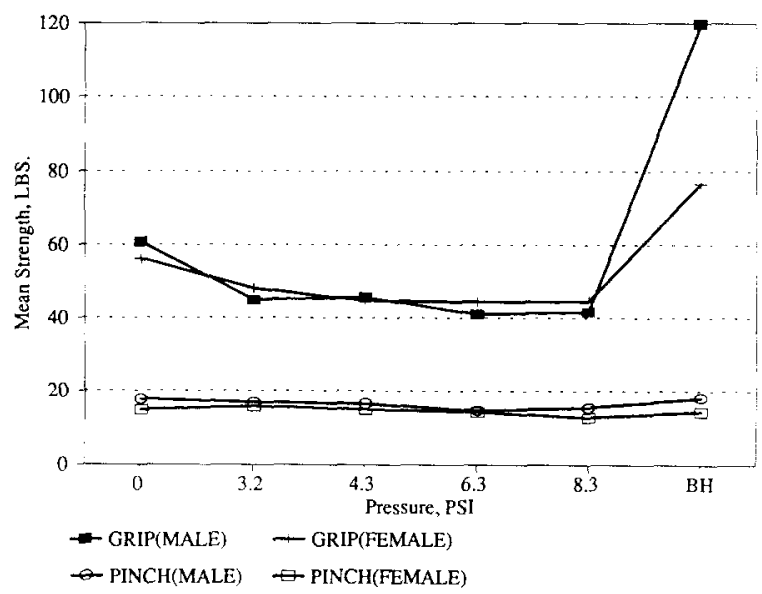

Fig. 4. Pressure effect on strength.

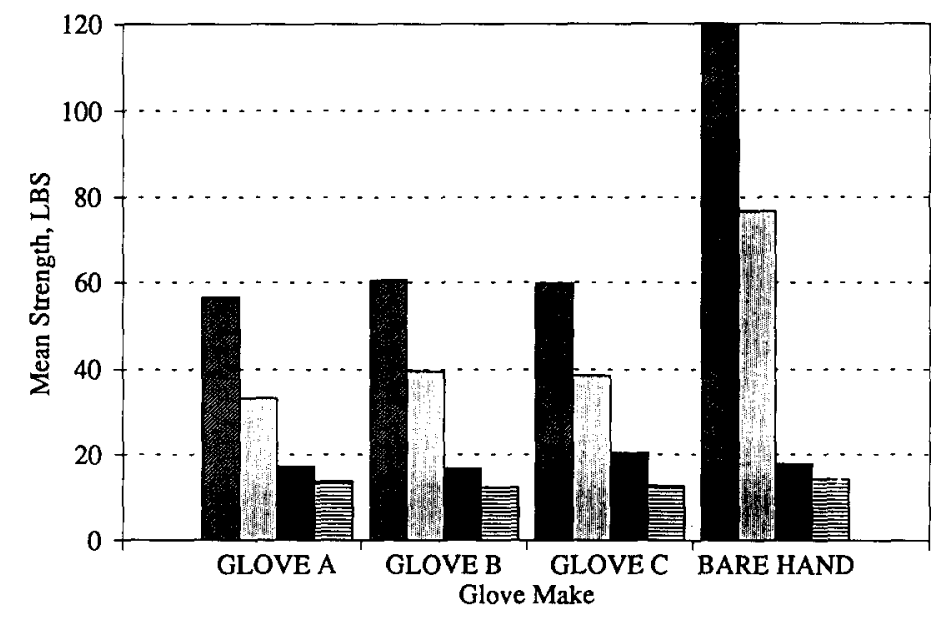

GRIP(MALE)

Fig. 3. Glove effect on strength. 


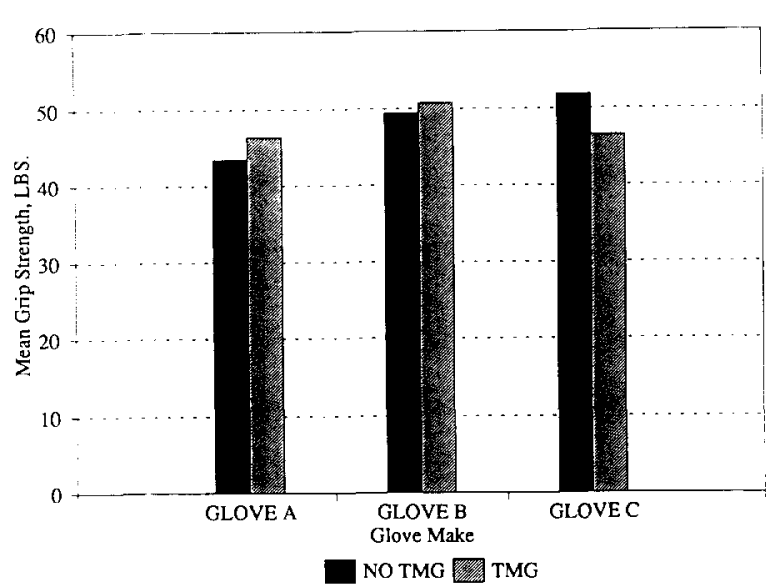

Fig. 5. Glove * TMG interaction.

exist (Fig. 6), with the female strength reducing with TMG, while the male strength increases with TMG. Size and extent of fit may be causing this result.

\subsection{Dexterity measures as dependent variable}

An analysis of variance (ANOVA) was performed on the data for all the dependent measures. The ANOVA summary is given in Table 4.

It is seen that the Gender effect, Glove effect, TMG effect, and the Pressure effect are significant for almost all of the dexterity variables, while there appears to be a significant Gender

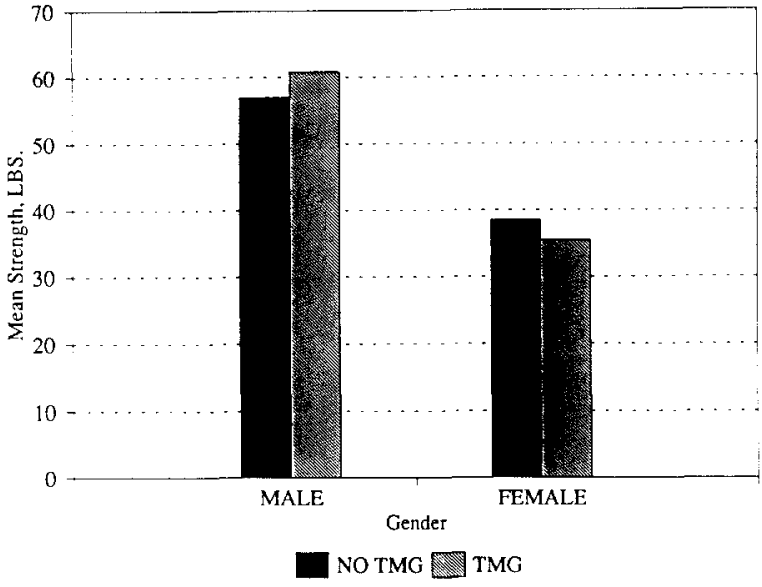

Fig, 6. TMG * gender interaction.

and TMG effect for tactility, as measured by the two-point discrimination test. Table 5 shows the summary of the Gender effect for the various dependent measures. It is noted that the female subjects were significantly slower than the male subjects. Fig. 7 shows the plot of the Glove effect on the mean nut and bolt assembly time. It is interesting to note that the performance with gloves is nearly 5 to 6 times slower than barehanded performance. Fig. 8 shows the plot of the pressure effect on the nut and bolt assembly time. The performance decreases with increasing pressure, and that increase is not uniform. A size effect appears to be present with smaller size

Table 4

Summary of ANOVA for the dexterity measures

\begin{tabular}{|c|c|c|c|c|c|c|c|}
\hline Effects & $\begin{array}{l}\text { Small } \\
\text { nut } \\
\text { and } \\
\text { bolt }\end{array}$ & $\begin{array}{l}\text { Medium } \\
\text { nut } \\
\text { and } \\
\text { bolt }\end{array}$ & $\begin{array}{l}\text { Large } \\
\text { nut } \\
\text { and } \\
\text { bolt }\end{array}$ & $\begin{array}{l}\text { Small } \\
\text { knot }\end{array}$ & $\begin{array}{l}\text { Medium } \\
\text { knot }\end{array}$ & $\begin{array}{l}\text { Large } \\
\text { knot }\end{array}$ & TPD \\
\hline Gender & $* * *$ & $* * *$ & $* * *$ & $* * *$ & *** & $* * *$ & $* * *$ \\
\hline Subject & $* * *$ & $* * *$ & $* * *$ & $* * *$ & $* * *$ & $*$ & $* * *$ \\
\hline Glove & ns & $* * *$ & ns & ns & ns & $\mathrm{ns}$ & $\mathrm{ns}$ \\
\hline TMG & ns & $* * *$ & $\mathrm{~ns}$ & $\mathrm{~ns}$ & $* *$ & $\cdots$ & $* * *$ \\
\hline Psi & $* * *$ & $* * *$ & $* * *$ & $* * *$ & $* * *$ & $\cdots$ & ns \\
\hline Gender * glove & ns & $* * *$ & ns & $* *$ & $*$ & $*$ & * \\
\hline Gender * TMG & $*$ & ns & ns & ns & ns & ' & * \\
\hline Gender * psi & ns & $* * *$ & ns & ** & ns & $*$ & ns \\
\hline Glove * TMG & $* *$ & $* *$ & ns & $*$ & $*$ & * & ns \\
\hline Glove * psi & $* *$ & ns & ns & $* *$ & ** & + & ns \\
\hline $\mathrm{TMG} * \mathrm{psi}$ & $* *$ & $* * *$ & ns & * & $*$ & * & $\mathrm{ns}$ \\
\hline
\end{tabular}


Table 5

Mean time for males and females

\begin{tabular}{lcc}
\hline Performance measure & $\begin{array}{l}\text { Mean time } \\
\text { males }\end{array}$ & $\begin{array}{l}\text { Mean time } \\
\text { females }\end{array}$ \\
\hline $\begin{array}{l}\text { Small nut and bolt } \\
\text { assembly time }\end{array}$ & $65.93 \mathrm{sec}$. & $94.45 \mathrm{sec}$. \\
$\begin{array}{l}\text { Medium nut and bolt } \\
\text { assembly time }\end{array}$ & $54.52 \mathrm{sec}$. & $83.13 \mathrm{sec}$. \\
$\begin{array}{l}\text { Large nut and bolt } \\
\text { assembly time }\end{array}$ & $45.63 \mathrm{sec}$. & $70.24 \mathrm{sec}$. \\
$\begin{array}{l}\text { Small knot tying time } \\
\text { Medium knot tying } \\
\text { time }\end{array}$ & $86.91 \mathrm{sec}$. & $117.28 \mathrm{sec}$. \\
$\begin{array}{l}\text { Large knot tying time } \\
\text { Two-point } \\
\text { discrimination length }\end{array}$ & $50.26 \mathrm{sec}$. & $117.28 \mathrm{sec}$. \\
\hline
\end{tabular}

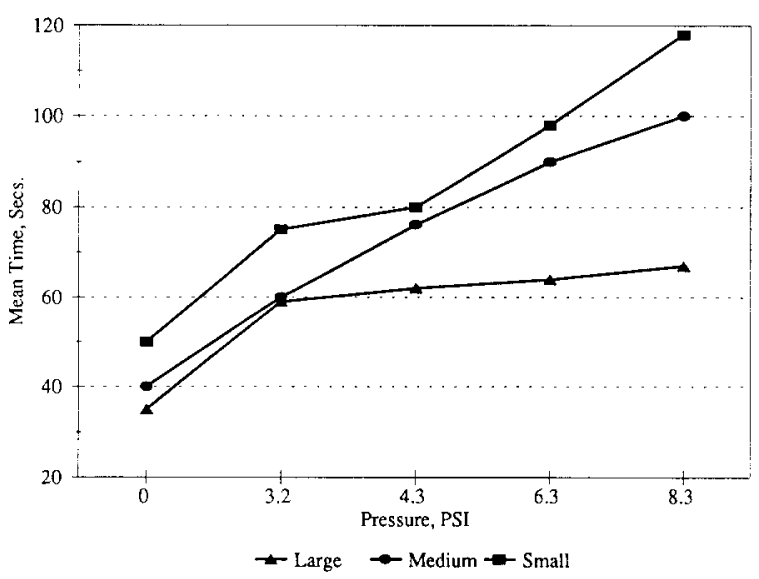

Fig. 8. Pressure effect on nuts and bolts assembly time.

taking longer time. The Glove effect on the mean knot-tying time is shown in Fig. 9. Again, as in the case of nut and bolt assembly time, the difference between gloved performance and barehanded performance is large. Fig. 10 shows the plot of the Pressure effect on the knot-tying time. The performance seems to decrease with increasing pressure. The decrease is not uniform. Two distinct patterns are seen, one between 0 psi and 3.2 psi, while the other between 3.2 psi and 8.3 psi. Fig. 11 shows the plot of the Glove * TMG interaction on the mean medium knot-tying time. Gloves A and B appear to be better than Glove C.

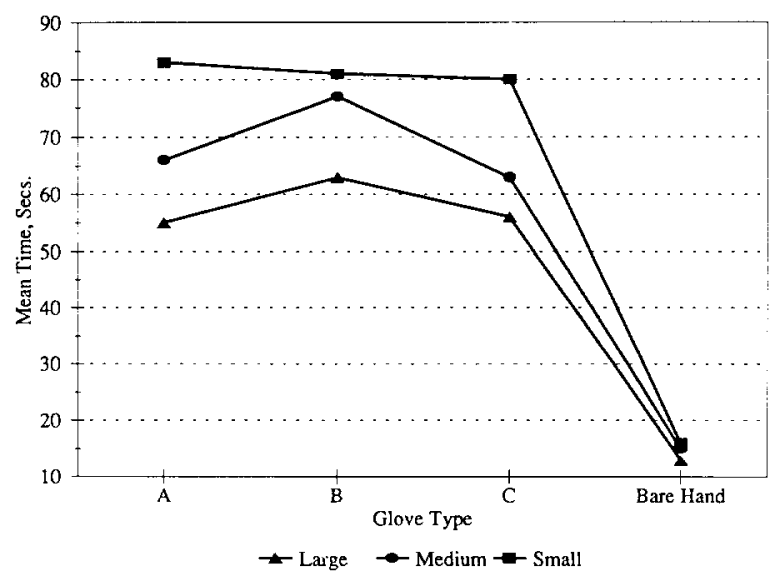

Fig. 7. Glove effect on nuts and bolts assembly time.

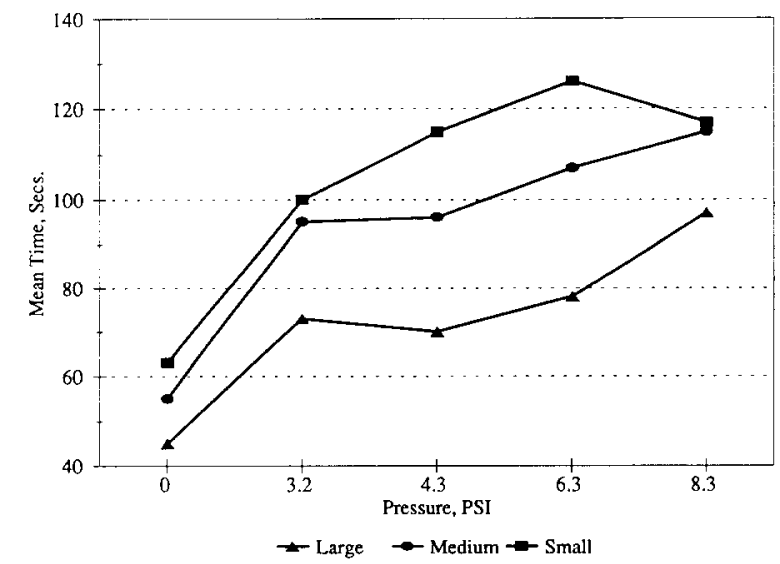

Fig. 10. Pressure effect on knot-tying time.

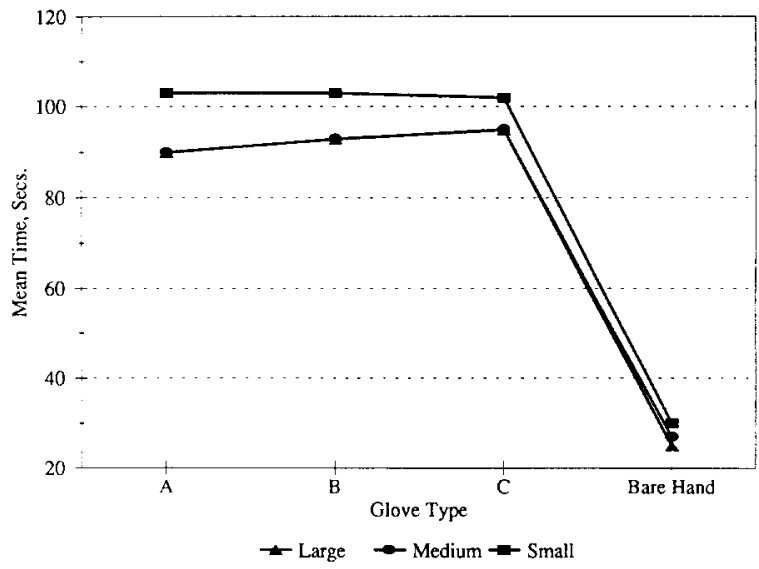

Fig. 9. Glove effect on knot-tying time. 


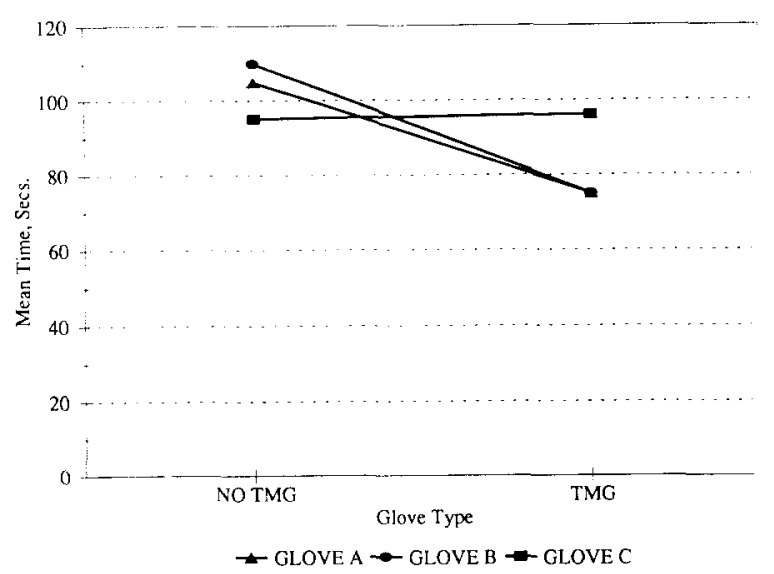

Fig. 11. Glove * TMG interaction on medium knot-tying time.

\subsection{Two-point discrimination test results}

An interesting finding of this investigation was that while tactile performance decreased with TMG, performance in knot-tying and nut-bolt assembly tasks improved with TMG. Either the two-point discrimination test was inadequate, or there is something more to the relationship between tactility and dexterity than what was being measured through a $2 \mathrm{PD}$ test here.

\section{Discussion and conclusion}

The Gender effect was perhaps the most consistent finding of this experiment. Female subjects tended to perform slower, and showed lower strength capabilities. Tables 2 and 5 show the mean time for male and female subjects for the different dependent measures.

The next major finding of this experiment is that both pressure and glove reduce performance. It is also apparent that gender differences are more defined based on both bare-handed and gloved conditions at zero psi differential, than at other conditions. These findings are consistent with those reported by O'Hara et al. (1988) and others (Wang et al., 1987; Cochran et al., 1986). With gloves there is an apparent increase in grip span, and an earlier pressing of fingers with each other. The former should increase the grip strength, while the latter should reduce the strength. It appears that the effects of increase in grip span with gloves is somewhat counteracted by the reduction in the inter-digital movements and range of motion when gloves are donned, resulting in net reduction in performance. Some of the observed gender differences may also have been due to lack of fit between hand and glove. Lack of glove effect on pinch strength is consistent with the results reported by Hallbeck and McMullin (1991). As the points of application of pinch force are at the tips of digits 1,2 and 3 a glove effect was not expected. In fact gloves may even increase pinch force due to the extra cushioning provided at the point of contact.

The reduced performance on dexterity measures with gloves is perhaps due to reduced range of motion and tactile sensitivity. With gloves one would expect reduced inter-digital movements, range of motion, and tactile sensitivity. These were perhaps causing the observed performance decrements. Although the performance with gloves was reduced as compared to the barehanded condition, the respective performances among the three gloves tested were comparable. An interesting finding of this investigation was that while tactile performance decreased with TMG, performance in knot-tying and nut-bolt assembly tasks improved with TMG. Either the 2PD test was inadequate, or there is something more to the relationship between tactility and dexterity than what was being measured through a 2PD test here.

One of the objectives of this experiment was to perform a comparison of the three gloves, with and without TMG. An explanation for TMG is in order here. Space Shuttle gloves have two components, an inner glove which has all the hardware for pressurization, and an outer glove to protect the wearer from the harsh thermal micrometeoroid environment of the outer space. The outer glove is called TMG, and was one of the factors investigated here. A possible glove * TMG interaction can have some interesting implications for the designers. The TMG of glove $\mathrm{B}$ appears to be the best, while that of glove $\mathrm{C}$ is the worst. The results suggest that in case of glove C TMG does 
not change the performance level, while it does offer the needed protection. However, the TMG of gloves $\mathrm{B}$, and $\mathrm{A}$, in addition to the providing protection against environment seem to improve performance as well. Overall, glove B seems to be the best. Its TMG shows the best performance improvement, and it has the best strength performance at all the pressure differential. Its dexterity performance, however, was comparable to that of glove A, and much better than glove C. Glove $B$ has a metacarpal joint as part of its design feature. Perhaps it is this difference that is causing it to perform best. More investigation is needed on this issue.

There were some other interesting interactions as well in this experiment. Male subjects' performance improved in the order $\mathrm{A}, \mathrm{B}$, and $\mathrm{C}$, while female subjects' performance improved in the opposite order C, B, and A.

In summary, it is seen that with gloves strength is reduced by nearly $50 \%$. Further performance decrements occur with increasing pressure differential, and TMG effects are not consistent across the three gloves tested. Size was not controlled in this study and may have had an impact on the findings. More research is needed to determine the exact effects of size and glove material on performance. Such a data will be invaluable to the designer of hand gloves.

\section{References}

Bishu, R.R. and Klute, G., 1993. Investigation of the effects of extra vehicular activity (EVA) gloves on performance. NASA Technical Paper no. 3401, Johnson Space Center, NASA, Houston, Texas.

Bradley, J.V., 1969a. Effect of gloves on control operation time. Human Factors, 11: 13-20.

Cochran, D.J., Albin, T.J., Bishu, R.R. and Riley, M.W., 1986. An analysis of grasp force degradation with commercially available gloves. Proceedings of the 30 th Annual Meeting of the Human Factors Society, 852-855.

Hallbeck, M.S. and McMullin, D., 1991. The effect of gloves, wrist position, and age on peak three-jaw pinch force: $A$ pilot study. In the Proceedings of the 35th Annual Meeting of the Human Factors Society, Santa Monica, CA, pp. 753-757.

Lyman, J., and Groth, H., 1958. Prehension force as a measure of psychomotor skill for bare and gloved hands. Journal of Applied Psychology, 42 (1): 18-21.

O'Hara, J.M., Briganti, M., Cleland, J. and Winfield, D., 1988. Extravehicular Activities Limitations Study. Volume II: Establishment of Physiological and Performance Criteria for EVA Gloves-Final Report (Report number ASEVALS-FR-8701, NASA Contract no NAS-9-17702.

Wang, M.J., Bishu, R.R. and Rodgers, S.H., 1987. Grip strength changes when wearing three types of gloves. Proceedings of the Fifth Symposium on Human Factors and Industrial Design in Consumer Products, Interface 87, Rochester, NY. 